\title{
Spontaneous telangiectatic osteosarcoma in a rhesus macaque (Macaca mulatta)
}

\author{
B. Goldschmidt ${ }^{1}$ (D) | M.I.Z. Calado ${ }^{1}$ | F.C. Resende ${ }^{1}$ | R.M. Caldas ${ }^{2}$ | L.W. Pinto ${ }^{3}$ | \\ C.A.A. Lopes $^{1}$ | F.G.O. França ${ }^{4}$ | B.S. Meireles ${ }^{1}$ | I.V. Souza ${ }^{1}$
}

${ }^{1}$ Serviço de criação de primatas não humanos, Instituto de Ciência e Tecnologia em Biomodelos (ICTB), Fundação Oswaldo Cruz (FIOCRUZ), Rio de Janeiro, Brazil

${ }^{2}$ Laboratório de Pesquisa Clínica em Dermatozoonoses em Animais Domésticos, Instituto Nacional de Infectologia Evandro Chagas, Fundação Oswaldo Cruz, Rio de Janeiro, RJ, Brazil

${ }^{3}$ Divisão de Patologia, Instituto Nacional de Câncer, Rio de Janeiro, RJ, Brazil

${ }^{4}$ Setor de radiologia, Nacional de Infectologia Evandro Chagas, Fundação Oswaldo Cruz, Rio de Janeiro, RJ, Brazil

\section{Correspondence}

Beatriz Goldschmidt, Serviço de criação de primatas não humanos, Instituto de Ciência e Tecnologia em Biomodelos (ICTB), Fundação Oswaldo Cruz (FIOCRUZ), Avenida Brasil, Rio de Janeiro, Brazil.

Email: bibi@fiocruz.br

\begin{abstract}
Osteosarcoma (OS) is the most common type of bone cancer, especially in young. Telangiectatic osteosarcoma (TO) is a rare variant of OS, and hence, its occurrence, presentation, and prognosis are poorly understood. A 4-year-old female rhesus monkey presenting lameness and swelling was examined for a mass on the right humerus. Radiography revealed fracture and disorganized structure of bone tissue. Histopathological examination revealed malignant neoplasm composed of anaplastic osteoblasts, which invaded the bone marrow and surrounded blood-filled cysts in the epiphysis and diaphysis forming septa. Cytogenetic analysis showed aneuploid cells, supernumerary AgNORs, and a marker fragment. The neoplasm was diagnosed as TO. To our knowledge, the occurrence of TO and its cytogenetic analysis were reported for the first time in non-human primates.
\end{abstract}

KEYWORDS

bone cancer, chromosomes, non-human primate, osteosarcoma

\section{1 | INTRODUCTION}

Osteosarcoma (OS) is the most common primary malignant bone tumor in humans, dogs, and cats. ${ }^{1}$ In non-human primates, spontaneous OS is rarely described and only few cases can be found in the literature in rhesus macaques (Macaca mulatta). ${ }^{2-4}$

Telangiectatic osteosarcoma (TO) is one of the rare subtypes of OS. It accounts for about 3\%-10\% of all human OSs with a preponderance of males and occurs mainly in the younger age group. The most common location at presentation is the metaphyses of long bones. The distal femur is involved most frequently, followed in frequency by the proximal tibia and proximal humerus. ${ }^{5}$ This type of OS has not been described in non-human primates. Although conventional OSs may contain telangiectatic elements, only those composed almost entirely of telangiectatic tissue are generally considered true examples of this entity. The most common and most important differential diagnostic consideration is aneurismal bone cyst ( $A B C)$. The radiographic and gross appearances of TO and $A B C$ show varying similarities. ${ }^{5}$ Another important diagnostic consideration includes giant cell tumor of bone. The histologic appearances of these entities are very different and are easily distinguishable from TO. The presence of vascular spaces lined only by endothelial cells and the absence of osteoid in the hemangiosarcoma are the key histologic features used to differentiate hemangiosarcoma from TO that needs to be considered in the differential diagnosis for a lytic bone tumor that appears to be malignant. Telangiectatic osteosarcoma is an aggressive tumor that destroys pre-existing bone and forms blood-filled cysts lined by malignant osteoblasts that form septae, with or without production of osteoid. ${ }^{1,5}$ Radiographically, TO appears as purely lytic destructive lesions mainly located in the metaphyses and affecting the medullary and cortical bones, with poorly demarcated margins. ${ }^{5}$ The exact cause of TO is unknown; however in humans, the familial occurrence in cousins suggests a genetic participation. ${ }^{6}$ The definition of alterations has increased from visualization at the chromosome level to point with OS mutations, but the genetic etiology of OS is still unknown. ${ }^{7}$ Conventional cytogenetic studies have demonstrated that OS exhibits high karyotype heterogeneity, with different degrees of aneuploidy and complex structural rearrangements. Although many cytogenetic 
abnormalities are associated with OS, a specific cytogenetic diagnostic or molecular marker has not been identified. ${ }^{5}$

\section{2 | MATERIAL AND METHODS}

The rhesus macaques maintained in the Laboratory Animal Breeding Center from Fiocruz Foundation are provided with filtered treated water ad libitum and nourished with a commercial primate chow (Nuvilab Primatas Velho Mundo ${ }^{\circledR}$, Quimtia Nuvital, Colombo, PR, Brazil) in the morning, supplemented with fruits and vegetables in the afternoon, which first were immersed in a $2 \%$ sodium hypochlorite solution for parasitological and bacteriologic control. The enclosures are cleaned with brooms daily and washed with pressurized water three times each week; no chemical is applied. Each enclosure has environmental enrichment items and is located in natural lighting environment with no controlled ambient temperature. The breeding colony is maintained in compliance with Brazilian law and approved by the Ethics Commission on Animal Experimentation of the Oswaldo Cruz Foundation under protocol number LW5/16. A female identified as AF26, aged 4 years with a body weight of $5.240 \mathrm{~kg}$, showed swelling and lameness of the right forelimb and difficulty in supporting weight. As soon as this was noted, she was caught for examination. During contention procedure, right humerus suffered a fracture and it was noticed an overrun in the proximal region of this arm. The animal was taken to the operating room, hand-injected with ketamine hydrocloride (Rhobifarma, Jd. Boa Vista, SP, Brazil) 12 mg/kg IM, midazolam (Medley Indústria Farmacêutica Ltda., Campinas-SP, Brazil) 0.2 mg/kg IM, and 0.5 mg fentanil (GlaxoSmithKline Manufacturing S.p.A., Pama, Italy) to perform radiography, collection of material for biopsy, and blood analysis. For cytogenetic, $2 \mathrm{~mL}$ blood from femoral vein was collected in heparinized syringe for lymphocyte culture and application of nucleolar organiser regions (AgNOR) staining technique on chromosomes. For hematic biometry, $0.5 \mathrm{~mL}$ was placed in tubes in which ethylenediaminetetraacetic acid (EDTA) was included as an anticoagulant. We estimated the concentration of six different constituents: hemoglobin concentration, hematocrit, red blood cells, mean corpuscular volume, mean corpuscular hemoglobin, and mean corpuscular hemoglobin concentration. All parameters were quantified with a Sysmex ${ }^{\circledR}$ Poch-100IV Diff equipment. For the blood biochemistry test, $2 \mathrm{~mL}$ of blood was emptied into a tube with a gel to separate it from the serum. We determined the concentration of fourteen different parameters: glucose, urea, total protein, creatinine, cholesterol, triglycerides, alkaline phosphatase, sodium, potassium, total bilirubin, alanine aminotransferase, lactate dehydrogenase, gammaglutamil transferase, and iron. These were quantified with a Johnson \& Johnson Vitros 250 Chemistry Analyzer (Johnson \& Johnson de México, S.A. de C.V.). Due to the advanced state of tumor, the animal was submitted to euthanasia performed by ketamine hydrocloride $(10 \mathrm{mg} / \mathrm{kg})+$ midazolam $(0.1 \mathrm{mg} / \mathrm{kg})$ followed by intravenous overdose of sodium pentobarbital (Tiopental - Cristália, São Paulo, Brazil) $30 \mathrm{mg} / \mathrm{kg}$. A sample of tumor tissue was collected with clamp and disclosed in RPMI culture medium for cytogenetic analysis. The body was sent to necropsy, and complete tissue sets were taken as indicated for histologic evaluation. Samples collected from the right humerus containing the lesion, kidneys, lungs, liver, spleen, mesenteric lymph nodes, and pancreas were fixed in $10 \%$ buffered formalin and processed routinely for embedding in paraffin. Bone sections were decalcified in $10 \%$ nitric acid. Then, all these tissue samples were processed routinely for embedding in paraffin, sectioned at $5 \mu \mathrm{m}$, and stained with hematoxylin-eosin (HE).

\section{3 | RESULTS}

Anteroposterior radiograph of the affected humerus revealed a disorganized structure of bone tissue with lytic destructive lesion in almost the entire bone, involving the metaphyseal region and extending to the proximal epiphysis leading to suspected OS. The fractured segments can be observed in the bone ends (Figure 1). Hematic biometry revealed mild anemia (4.35 million $/ \mathrm{m}^{3}$ erythrocytes while reference value is $5.062 \pm 0.539$ and $9.50 \mathrm{~g} / \mathrm{dL}$ hemoglobin while reference value is $12.76 \pm 1.097$ ). Alkaline phosphatase was in physiological range $(196 \mathrm{U} / \mathrm{L})$. Serum iron test showed a very low value of $13 \mathrm{mg} / \mathrm{dL}$, while the reference value is $130-190 \mathrm{mg} / \mathrm{dL}$ (Table 1). In the necropsy, the gross lesions were a dark red mass with a spongy-like appearance that filled the medullary cavity of the fragments of the lytic right humerus (Figure 2), multiple dark red nodules measuring between 0.7 and $1.5 \mathrm{~cm}$ on the liver surface (Figure 3 ) that extended to parenchyma and one dark red nodule measuring $0.5 \mathrm{~cm}$ in diameter in the parenchyma of a mesenteric lymph node. No metastases were found in the lung. Bone sections were decalcified in $10 \%$ nitric acid. Then, all these tissue samples were processed routinely for embedding in paraffin, sectioned at $5 \mu \mathrm{m}$, and stained with $\mathrm{HE}$. Histopathological examination of the right humerus revealed malignant neoplasm composed of anaplastic osteoblasts, which invaded the bone marrow and surrounded blood-filled cysts in the epiphysis and diaphysis forming septa (Figures 4 and 5). Sometimes, there were large solid tumor masses. The nuclei of the neoplastic cells were pleomorphic, oval to elongated, central, and hyperchromatic (Figure 4). There was gigantism of nucleoli, which sometimes reached the number of two per cell. The cytoplasm of neoplastic cells was abundant, elongated, eosinophilic, and with indistinct borders (Figure 4). There was osteoid production in small amounts (Figure 5). There were one to three mitotic figures per high power field (400x), and some of them were atypical. Multinucleated giant cells were observed in the septae of neoplastic cells or within the blood-filled cysts. Liver and mesenteric lymph nodes presented metastasis of the tumor described in the humerus. In the right kidney, there was a multifocal and discreet chronic interstitial nephritis. The inflammatory infiltrate was composed mainly of lymphocytes and had also plasmocytes and macrophages. In the left kidney, spleen, pancreas, and lungs, there were no histologic alterations. The conventional cytogenetic analysis of 30 metaphases collected directly from tumor, which featured a sponge-like appearance, revealed the most frequent chromosomal alterations as a marker supernumerary chromosome. This fragment was present in aneuploid cells with too 


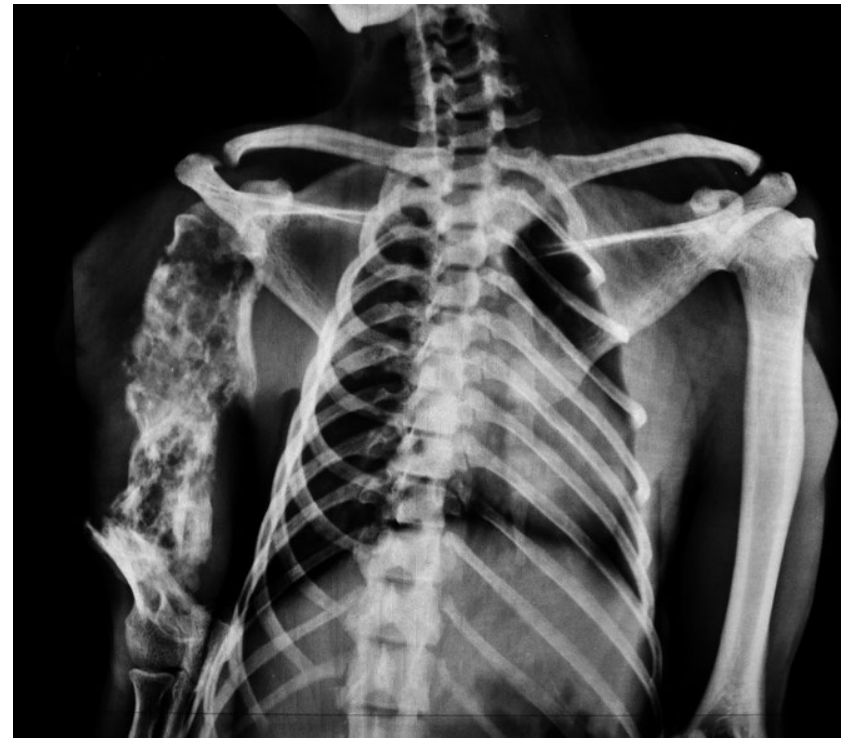

FIGURE 1 Telangiectatic osteosarcoma. Rhesus macaque. Radiographic projection showing disorganized structure of bone tissue with lytic destructive lesion in almost the entire right humerus and fractures near epiphyseal

many chromosomes or less. The AgNOR staining technique showed more than two chromosomes carrying these sequences in tumor cells (Figure 6). Originating metaphases from peripheral blood lymphocytes did not show the chromosomal fragment nor aneuploidy and only two AgNORs-colored regions.

\section{4 | DISCUSSION}

Although related to more preponderance in human males ${ }^{5}$ and occurrence among cousins, ${ }^{6}$ TO in this case affected a female without other family cases. According to its histologic features and based on the World Health Organization classification system, ${ }^{1}$ the neoplasma in the right humerus was diagnosed as TO with liver and mesenteric lymph node metastasis. The radiographic, gross, and histologic findings were similar to those described for TO in humans, ${ }^{5}$ dogs, ${ }^{8}$ cats, ${ }^{9}$ and horses, ${ }^{10}$ showing a very aggressive behavior. The anemia detected and low value of serum iron may reflect blood loss from the tumor. Metastases observed in this study are probably from the TO which easily metastasizes and is highly fatal. ${ }^{1}$ In the tumor described in this study, the presence of malignant osteoblasts instead of endothelium lining the blood-filled cysts, the production of osteoid, and the presence of a few number of giant multinucleate cells ruled out hemangiosarcoma, $A B C$, and giant cell tumor of bone, which are considered the main differential diagnosis of TO. ${ }^{1,5,8}$ It was evident from the earliest studies of ploidy in OSs that these tumors showed a significant propensity toward aneuploidy. ${ }^{11-14}$ Cytogenetic findings suggest implication of the chromosome fragment in tumoral process and an increase in protein synthesis represented by the increase in ribosomal RNA transcription activity. The number of silver-staining nucleolar organized regions (AgNOR) per nucleus was significantly higher offering
TABLE 1 Values of hematic biometry and blood chemistry for the rhesus Female AF26

\begin{tabular}{|c|c|}
\hline Results & Reference values $^{17}$ \\
\hline \multicolumn{2}{|l|}{ Hematic biometry } \\
\hline Blood count (RBC) 4.3 millions $/ \mathrm{mm}^{3}$ & $5.062 \pm 0.539$ \\
\hline Hemoglobin dosage (HGB) $9.5 \mathrm{~g} / \mathrm{dL}$ & $12.76 \pm 1.097$ \\
\hline Hematocrit (Hct) 33\% & $37.55 \pm 3.23$ \\
\hline Mean corpuscular volume (MCV) $75.90 \mathrm{fm}^{3}$ & $74.46 \pm 5.11$ \\
\hline $\begin{array}{l}\text { Mean corpuscular hemoglobin }(\mathrm{MCH}) \\
21.80 \mathrm{pg}\end{array}$ & $25.34 \pm 1.74$ \\
\hline $\begin{array}{l}\text { Mean corpuscular hemoglobin concentra- } \\
\text { tion }(\mathrm{MCHC}) 28.80 \mathrm{~g} / \mathrm{dL}\end{array}$ & 34 \\
\hline Platelet count $348000 / \mathrm{mm}^{3}$ & $359000 \pm 71.72$ \\
\hline Leukocyte count (WBC) $9000 / \mathrm{mm}^{3}$ & $7.890 \pm 3.53$ \\
\hline \multicolumn{2}{|l|}{ Blood chemistry } \\
\hline Sodium 149 mEq/L & $137-150$ \\
\hline Potassium $3.80 \mathrm{mEq} / \mathrm{L}$ & $3.20-4.30$ \\
\hline Glucose 66 mg/dL & $50-100$ \\
\hline Urea $36.40 \mathrm{mg} / \mathrm{dL}$ & $9-26$ \\
\hline Creatinine $0.70 \mathrm{mg} / \mathrm{dL}$ & $0.30-1.30$ \\
\hline Total bilirubin $0.10 \mathrm{mg} / \mathrm{dL}$ & $0.10-0.60$ \\
\hline Alanine aminotransferase $14 \mathrm{U} / \mathrm{L}$ & $18-129$ \\
\hline Lactate dehydrogenase $1104 \mathrm{UI} / \mathrm{L}$ & $578-1800$ \\
\hline Alkaline phosphatase $196 \mathrm{U} / \mathrm{L}$ & $65-400$ \\
\hline Gamma-GT $69 \mathrm{U} / \mathrm{L}$ & $48-130$ \\
\hline Triglycerides $55 \mathrm{mg} / \mathrm{dL}$ & $23-180$ \\
\hline Cholesterol 126 mg/dL & $73-204$ \\
\hline Iron $13 \mathrm{mg} / \mathrm{dL}$ & $130-190$ \\
\hline Total protein $7.10 \mathrm{~g} / \mathrm{dL}$ & $5.80-7.40$ \\
\hline
\end{tabular}

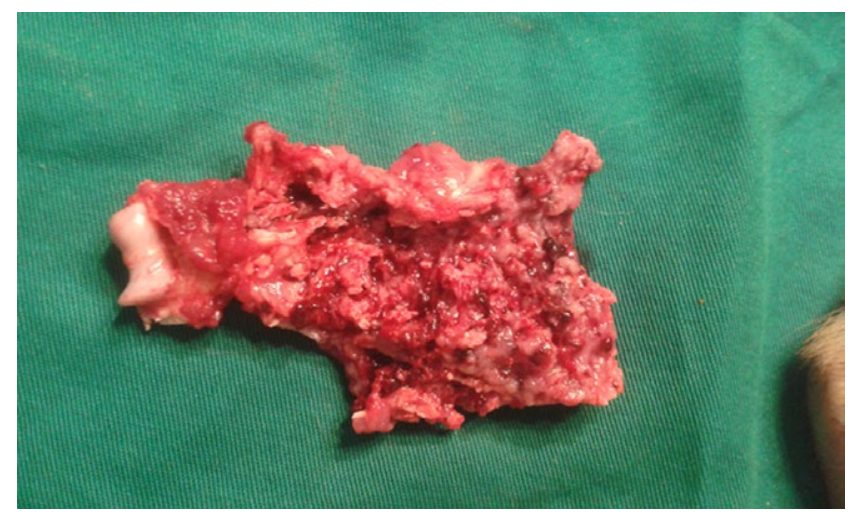

FIGURE 2 Telangiectatic osteosarcoma. The entire lesion was a dark red mass with a spongy-like appearance filled the medullary cavity of the right humerus

an advantage in differential diagnosis with non-malignant events. Mutations or deregulation of important genes for mitotic checkpoints is thought to be the underlying cause of chromosome instability. Areas of gene amplification, such as ring chromosomes, double minutes, and homogeneously staining regions, are fairly common in conventional 


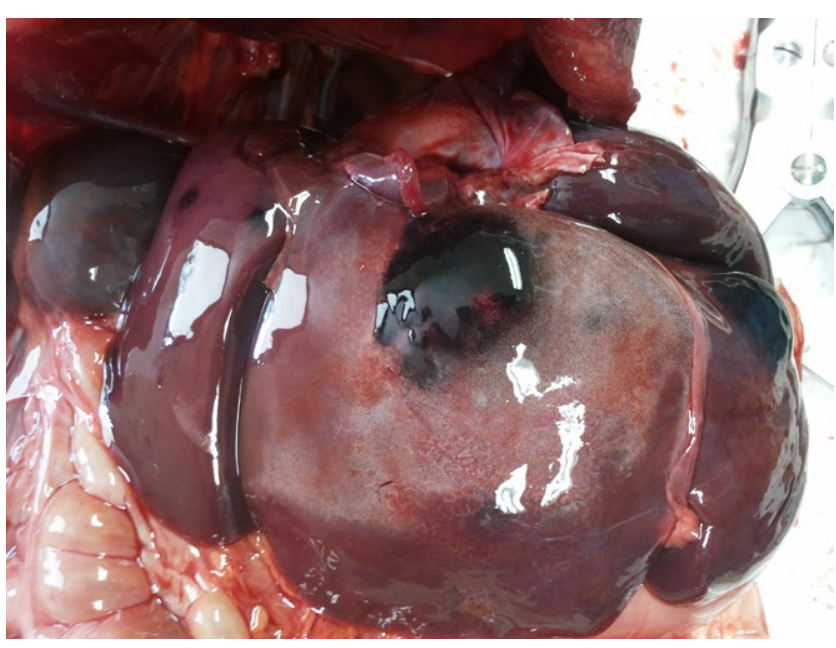

FIGURE 3 Telangiectatic osteosarcoma. Liver, rhesus macaque. Multiple dark red subcapsular nodules

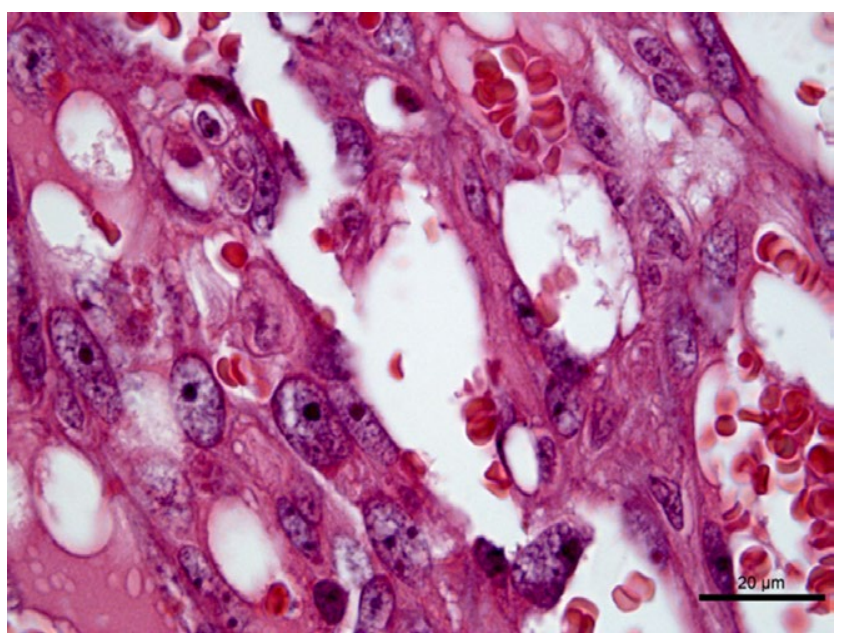

FIGURE 4 Telangiectatic osteosarcoma. Rhesus macaque. Malignant osteoblasts lining blood-filled cysts and showing nuclei pleomorphic, oval or elongated shape, central, and hyperchromatic and nucleolar gigantism. The cytoplasm of neoplastic cells is abundant, elongated, eosinophilic, and with indistinct borders. $\mathrm{HE}$, hematoxylin-eosin

OSs. ${ }^{15,16}$ Important strides are just now beginning to be made in identifying abnormalities that have prognostic and therapeutic implications. Although no standard recommendations for chemotherapy in TO exist, identification of OS cell type is crucial for characterizing genetic events prior to its full-blown clinical manifestation, which could provide insights into the choice of surgery and therapeutic approach. To our knowledge, the occurrence of TO and its cytogenetic analysis are reported for the first time in non-human primates.

\section{ACKNOWLEDGMENTS}

We thank the animal care staff of the Department of Primatology, Center for Laboratory Animal Breeding (ICTB), and Fiocruz and Ricardo Baptista Schmidt from Oswaldo Cruz Institute (IOC), Fiocruz, for processing the figures.

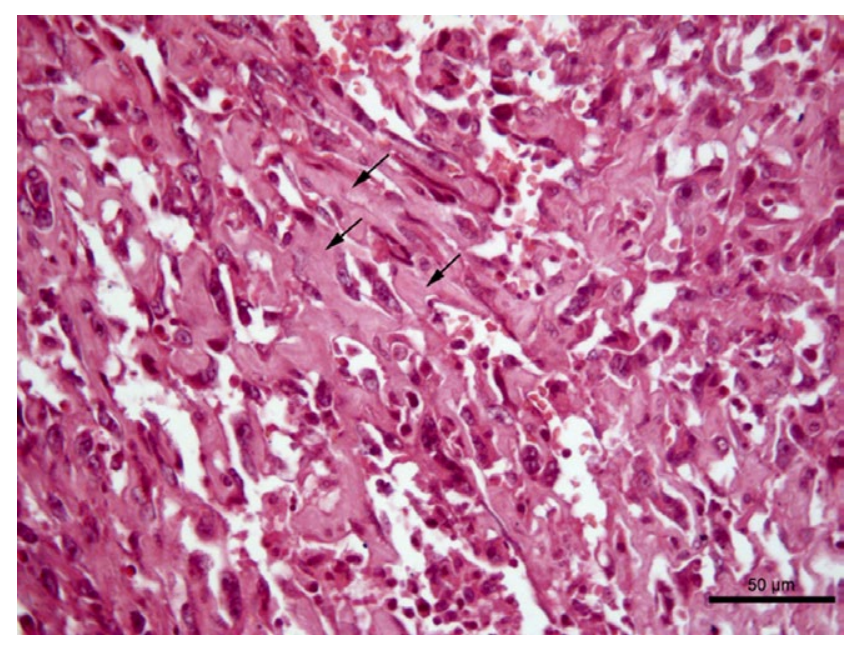

FIGURE 5 Telangiectatic osteosarcoma. Rhesus macaque. The presence of osteoid (arrows) in the septae formed by malignant osteoblasts that surround blood-filed cysts. HE, hematoxylin-eosin

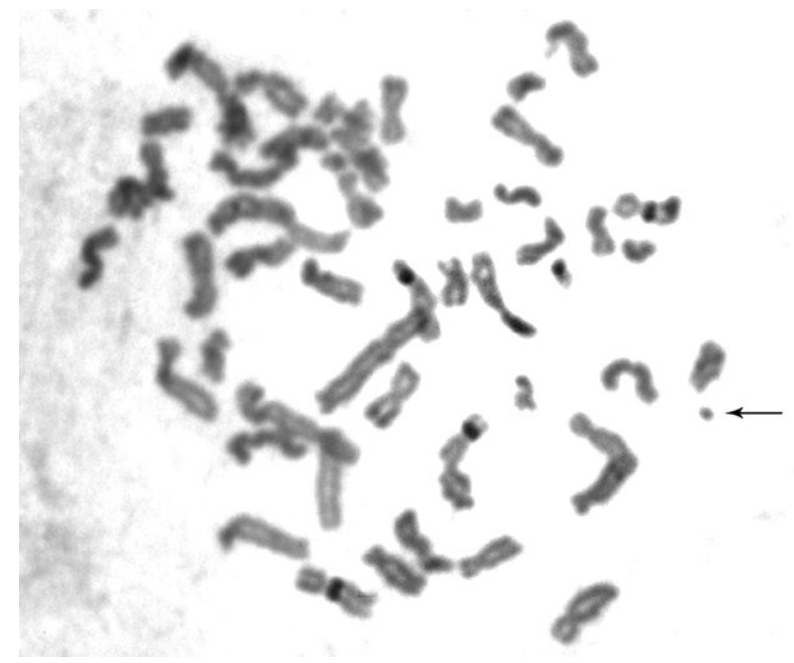

FIGURE 6 Telangiectatic osteosarcoma. Rhesus macaque. AgNOR staining technique showing more than two chromosomes carrying these sequences in tumor cells. Arrow indicates a chromosome fragment present in tumor cells

\section{CONFLICT OF INTEREST STATEMENT}

The authors declare that there are no potential conflict of interests related to the research or the authorship and/or publication of this article.

This research did not receive any specific grant from funding agencies in the public, commercial, or not-for-profit sectors.

\section{REFERENCES}

1. Slayter MV, Boosinger TR, Pool RR, Dämmarich K, Misdorp W, Larsen S. Histological Classification of Bone and Joint Tumors of Domestic Animals. World Health Organization. 2nd series, , vol. 1. Washington, DC: Armed Forces Institute of Pathology; 2007

2. Beam SL. Combined-type Osteosarcoma in a Rhesus Macaque. Vet Pathol. 2005;42:374-377. 
3. Seibold HR, Wolf RH. Neoplastic and proliferative lesions in 1065 nonhuman primate necropsies. Lab Anim Sci. 1973;23:533-539.

4. Sembrat F, Fritz GR. Long bone osteosarcoma in a rhesus monkey. J Am Vet Med Assoc. 1979;175:971-974.

5. Sangle NA, Layfield LJ. Telangiectatic Osteosarcoma. Arch Pathol Lab Med. 2012;136:572-576.

6. Nishida J, Abe M, Shiraishi $H$, et al. Familial occurrence of telangiectatic osteosarcoma: cousin cases. J Pediatr Orthop. 1994;14: 119-122.

7. Martin JW, Squire JA, Zielenska M. The Genetics of Osteosarcoma. Sarcoma 2012;2012:627254.

8. Brellou G, Papaioannou N, Patsikas M, Polizopoulou Z, Vlemmas I. Vertebral telangiectatic osteosarcoma in a dog. Vet Clin Pathol. 2004;33:159-162.

9. Havlicek M, O'Connell K, Langova V. Feline maxillary telangiectatic osteosarcoma in a two-year-old cat. Aust Vet Pract. 2008;38:92-96.

10. Gutierrez-Nibeyro SD, Sullins KE, Powers BE. Treatment of appendicular osteosarcoma in a horse. Equine Vet Educ. 2010;22: 540-544.

11. Bauer HC, Kreicbergs A, Silfversward C, Tribukait B. DNA analysis in the differential diagnosis of osteosarcoma. Cancer. 1988;61:1430-1436.

12. Hiddemann W, Roessner A, Wormann B, et al. Tumor heterogeneity in osteosarcoma as identified by flow cytometry. Cancer. 1987;59:324-328.
13. Kreicbergs A, Silvferswärd C, Tribukait B. Flow DNA analysis of primary bone tumors. Relationship between cellular DNA content and histopathologic classification. Cancer. 1984;53:129-136.

14. Kruzelock RP, Murphy EC, Strong LC, Naylor SL, Hansen MF. Localization of a novel tumor suppressor locus on human chromosome $3 q$ important in osteosarcoma tumorigenesis. Cancer Res. 1997;57:106-109.

15. Fletcher JA, Gebhardt MC, Kozakewich HP. Cytogenetic aberrations in osteosarcomas. Nonrandom deletions, rings, and double-minute chromosomes. Cancer Genet Cytogenet. 1994;77:81-88.

16. Sinovic JF, Bridge JA, Neff JR. Ring chromosome in parosteal osteosarcoma: clinical and diagnostic significance. Cancer Genet Cytogenet. 1992;62:50-52.

17. Andrade MCR, da Ribeiro CT, Silva VF, et al. Biologic data of Macaca mulatta, Macaca fascicularis, and Saimiri sciureus used for research at the Fiocruz Primate Center. Mem Inst Oswaldo Cruz. 2004;99:581-189.

How to cite this article: Goldschmidt B, Calado MIZ, Resende FC, et al. Spontaneous telangiectatic osteosarcoma in a rhesus macaque (Macaca mulatta). J Med Primatol. 2017;46:51-55. https://doi.org/10.1111/jmp.12254 